\title{
Oculomotor Nerve Disorder, CTCAE
}

National Cancer Institute

\section{Source}

National Cancer Institute. Oculomotor Nerve Disorder, CT CAE. NCI Thesaurus. Code C143711.

A disorder characterized by dysfunction of the oculomotor nerve (third cranial nerve). 